International Journal of Pure and Applied Mathematics

Volume 95 No. 3 2014, 347-356

ISSN: 1311-8080 (printed version); ISSN: 1314-3395 (on-line version)

url: http://www.ijpam.eu

doi: http://dx.doi.org/10.12732/ijpam.v95i3.3

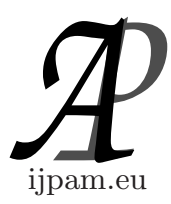

\title{
CONVEXITY AND TWO PIECE PROPERTY IN $S^{n}$
}

\author{
M. Beltgy ${ }^{1}$, S. Shenawy ${ }^{2}$, A. Elsharkawy ${ }^{3}$ § \\ ${ }^{1}$ Mathematics Department \\ Faculty of Science \\ Tanta University \\ Tanta, EGYPT \\ ${ }^{2}$ Modern Academy for Engineering and Technology in Maadi \\ Maadi, EGYPT \\ ${ }^{3}$ Faculty of Science \\ Tanta University \\ Tanta, EGYPT
}

\begin{abstract}
A subset $A$ of $S^{n}, n \geq 2$, has spherical two piece property if any hypersphere cuts $A$ into at most two pieces. A subset $A$ of $S^{n}$ has totally geodesic two piece property if any totally geodesic hypersurface in $S^{n}, n \geq$ 2 , cuts $A$ into at most two pieces. In this article we study some geometric properties of subsets in $S^{n}$-as a manifold with focal points and these types of two piece property in $S^{n}$.
\end{abstract}

AMS Subject Classification: 53C42, 52A05

Key Words: sphere $S^{n}$, convex sets, supporting totally geodesic hypersurface, spherical two piece property, totally geodesic two piece property and Tight immersions

\section{Introduction}

The notions of convexity and two piece property are very interesting, useful

Received: March 12, 2014

(C) 2014 Academic Publications, Ltd.

$\S_{\text {Correspondence author }}$ url: www.acadpubl.eu 
and have many applications in both geomerty and analysis. Convexity and two piece property have been generalized in many aspects and for different reasons. In this article we introduce two generalizations of two piece property in $S^{n}$, namely, spherical and totally geodesic two piece properties and we study some important related concepts in geomerty.

Alexander in [1] defined three different types of convexities:strong convexity, convexity and weak convexity in general Riemannian manifolds, for more details see [1]. Now, we take in consideration a strong convexity and this type is exactly coincident with the classical convexity concept mentioned later.

So, the classical concept of convexity of subsets in $S^{n}$ may be generalized as follows. A subset $A \subset S^{n}$ is said to be convex if for each pair of points $p, q \in A$, there is a unique minimal geodesic segment $[p q]$ joining $p, q$ in $S^{n}$ and this geodesic segment lies in $A$. For example,the geodesic ball $B(p, \delta) \subset S^{n}$ with center at $p$ and radius $\delta<\frac{\pi}{2}$ is convex, but the whole sphere $S^{n}$ is not convex.

In $E^{n}$ the closure of an open convex. In $S^{n}$ the closure of an open convex subset may not be convex. An example of this claim is the open hemispher in $S^{n}$.

We specialize now to talk about support element of subsets in $S^{n}$. For a subset $A$ of $S^{n}$, a totally geodesic hypersurface $T$ in $S^{n}$ is a supporting hypersurface of $A$ if $T$ intersects the closure of $A$ and a closed hemisphere generated by $T$ contains $A$. But $A$ has a local support element at $p$ if there exists a neighborhood $U$ about $p$ in $S^{n}$ such that $A \cap U$ is supported by a totally geodesic hypersurface $T$ at $p$.

Lemma 1. [1]. A connected open subset $A$ of $M$ is convex if and only if $A$ possesses a local support element at every boundary point and does not intersect its own cut locus $C(A)=\{p \in A$ : pisacutpointof someq $\in A\}$

A closed convex subset $A \subset S^{n}$ is called strictly convex if its boundary $\partial A$ contains no geodesic segments. A closed convex subset with a non-empty interior is called a convex body.

Lemma 2. Every closed geodesic ball with radius less than $\pi / 2$ in $S^{n}$ is a strictly convex body.

Definition 3. A point $p$ in a set $A \subset S^{n}$ is said to be an exposed point of $A$ if there exists a totally geodesic hypersurface $T$ that supports $A$ at $p$ and intersects $A$ only at $p$. The set of all exposed points of $A$ is denoted by $E_{x}(A)$. Also, a point $p \in A$ is called a local exposed point of $A$ if there exists a neighborhood $U$ of $S^{n}$ about $p$ such that $A \cap U$ has $p$ as an exposed point. 
Lemma 4. Let $A$ be a strictly convex subset of $S^{n}$ with smooth boundary. Then each boundary point is an exposed point.

For example, consider the geodesic ball $B(p, \delta) \subset S^{n}$ with center $p$ and radius $\delta<\frac{\pi}{2}$, all of its boundary points are exposed points but a closed hemisphere does not contain any exposed points. It is obvious that each exposed point must be a boundary point. Also, every global exposed point is a local one but the converse is not generally true. M. Beltagy [3] proved that, for a connected open subset $A \subset \widetilde{W}$, where $\widetilde{W}$ is a manifold without focal points, with smooth boundary $\partial A$. If each boundary point is a local exposed point of $\bar{A}$, then $A$ is a strictly convex body of $\widetilde{W}$. Also he introduced the relation between global exposed point and convexity as indicated in the following. For $A \subset \widetilde{W}$ be an open bounded subset with smooth boundary. If each $p \in \partial A$ is a global exposed point of $\bar{A}$, then $A$ is a strictly convex body of $\widetilde{W}$ [3].

Banchoff [2]started the subject of the two piece and spherical two piece properties for submanifolds of the Euclidean and Spherical two piece property in $E^{n}$. The study of the two piece and spherical two piece properties has been developed by other mathematicians [6, 7]. M. Beltagy [4] proved some properties in manifolds without focal points. We prepared a paper entiteled Convexity and Two piece property in $H^{n}$ as an example of a manifold witout focal points and we defined two types of the two piece property namely totally geodesic and horosphere two piece property and this paper is accepted for publication in international mathematical forum journal. The aim of this work is to extend and generalized the above mentioned study to include $S^{n}$ as a manifold with focal points.

Definition 5. A subset A of $S^{n}$ is said to have totally geodesic two piece property (in brief TTPP) if any totally geodesic hypersurface in $S^{n}$ cuts $A$ into at most two pieces. $A$ is said to have spherical two piece property (in brief $\mathrm{STPP}$ ) if any hypersphere cuts $A$ into at most two pieces.

Clearly any subset A of $S^{n}$ has STPP also has TTPP and the converse is not generally true according to the following example.

Let $A \subset S^{2}$ be the area bounded by two close meridians joining north and west poles $p$ and $q$. Let $\mathrm{C}$ be a geodesic circle with center $u$ as indicated in Figure 1. Clearly A has TTPP but does not have STPP.

Definition 6. A subset $A$ of $S^{n}$ with smooth boundary is said to be T-convex if $A$ is supported at each boundary point of $A$ by a totally geodesic hypersurface $T$.

It is known that the distance from a point $p$ to a non-empty subset $\mathrm{A}$ of 


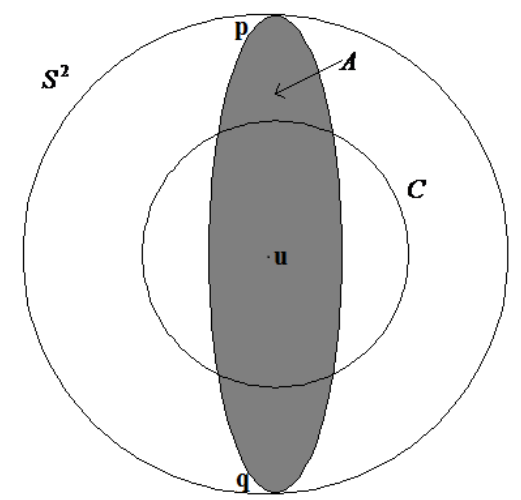

Figure 1: A has TTPP but does not have STPP.

Riemannien manifold $\mathrm{M}$ is defined as

$$
d(p, A)=\inf \{d(p, x): x \in A\}
$$

Definition 7. Let A be a subset of $S^{n}$. A point $p \in S^{n}$ has a farthest point $m \in A$ denoted by $F_{A}(p)=m$ if

1. $m \in A$.

2. $d(p, m) \geq d(p, x)$ for all $x \in A$.

Consider A to be a geodesic ball $B(q, r)$ with center $q$ and raduis $r \prec \pi / 2$. It is clear that $F_{A}(p)=q$ where $p$ is the antipodal point of $q$. This example shows that the farthest point may be an interior point. In $E^{n}$ the situation in $S^{n}$ is completely different.

Definition 8. Let A be a subset of $S^{n}$. A point $p \in S^{n}$ has a local farthest point $q \in A$ denoted by $F_{A}^{l}(p)=q$ if there exists a neighborhood $U$ of $q$ such that

1. $m \in A$.

2. $d(p, m) \geq d(p, x)$ for all $x \in A$.

Let us define the height function in $S^{n}$ with respect to a totally geodesic hypersurface $T$. Let $p$ be a center of $T$. For a subset A of $S^{n}$ we define a height function

$$
h_{T}^{p}: A \rightarrow R
$$


by

$$
h_{T}^{p}(x)=\pi / 2-d(p, x)
$$

for any $x \in A$

Note 9. In $S^{n}$ any totally geodesic hypersurface has two centers.

Geomertrically, $h_{T}^{p}$ is indicated in Figure 2. Clearly $h_{T}^{p}$ is a bounded function for each subset of $S^{n}$.

The properties of the height function $h_{T}^{p}(x)$ are smilar to that of the distance function $d(p, x)$.

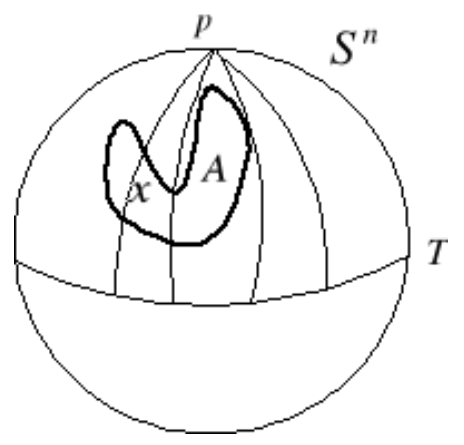

Figure 2

Definition 10. A subset $A$ of $S^{n}$ is said to be tight if each height function when restricted to $A$ has one strict local maximum at most.

\section{Results}

In this section we present the main results of this paper and we begin with the following lemmas.

Lemma 11. Any convex subset of $S^{n}$ has TTPP.

Proof. Suppose that a convex subset $A$ of $S^{n}$ does not have TTPP. Then there exists a totally geodesic hypersurface $T$ which cuts $A$ into more than two pieces. Without loss of generality suppose that $T$ cuts $\mathrm{A}$ into three pieces $A_{1}$, $A_{2}$ and $A_{3}$, then at least two of the pieces, say $A_{1}$ and $A_{2}$, lie on one side of $T$ see Figure 3. Let $p$ and $q$ be any two points one in each piece and lie in one side of $T$. For arbitrary points $p \in A_{1}$ and $q \in A_{2}$ the geodesic segment $[p q]$ 
which is minimal satisfies $[p q] \not \subset A$ which means that $A$ is not convex. This is a contradiction and the proof is complete.

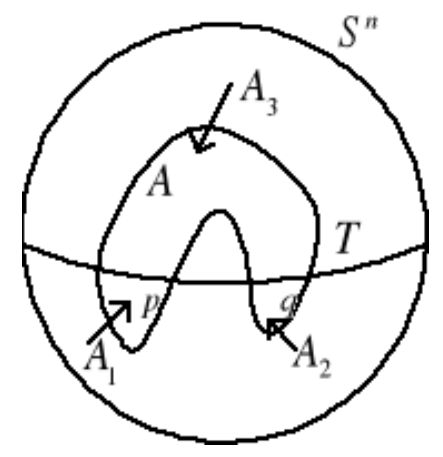

Figure 3

Remark 12. The converse of the above lemma is not generally true. For example the area between two concentric geodesic circles (annulus) in $S^{2}$ has TTPP but it is not convex.

Lemma 13. Let $A$ be a closed subset of $S^{n}$ with smooth boundary $\partial A$ and $\operatorname{int} A \neq \phi$. If $A$ is T-convex, then $A$ has TTPP.

Proof. Suppose that $A$ is T-convex. Then $A$ is supported at every boundary point by a totally geodesic hypersurface. Thus $\partial A$ has sectional curvature $K$ satisty $K \geq 1$.

Applying the following theorem due to Do Carmo [5] together with Lemma 11 we obtain that A has TTPP.

Theorem 14. Let $f: M \rightarrow S^{n+1}$ be an isometric immersion of a compact, connected orientable $C^{\infty} n$-dimensional Riemannian manifold $M$ into $(n+1)$-dimensional sphere $S^{n+1}$ of constant sectional curvature 1 and assume that all sectional curvatures $K$ of $M$ satisfy $K \geq 1$, then $f$ is an imbedding, $M$ is diffeomorphic with $S^{n}$, and $f(M)$ is either totally geodesic or contained in an open hemisphere. In the latter case $f(M)$ is the boundary of a convex body in $S^{n+1}$.

Remark 15. The converse of the above lemma is not generally true. Example given in Remark 12 indicates this claim.

Lemma 16. Let $A$ be a closed subset of $S^{n}$ with smooth boundary and int $A \neq \phi$. If each boudary point is exposed, Then $A$ has TTPP. 
Proof. Suppose that A does not have TTPP. Then there exists a totally geodesic hypersurface $T$ which cuts $A$ into more than two pieces. From Lemma $13 \mathrm{~A}$ is not $\mathrm{T}$-convex, i.e there exists a boundary point $p$ where $\mathrm{A}$ is not supported at it. Thus $p$ is not an exposed point, which is a contradiction.

Proposition 17. Let $A$ be a closed subset of $S^{2}$ having $S T P P$, and int $A \neq$ $\phi$. Let $p$ and $q$ be any two points in int $A$. If the minimal geodesic segment $[p q] \subset$ int $A$, then $A \backslash[p q]$ has $S T P P$.

Proof. Suppose that $A \backslash[p q]$ does not have STPP, then there exists a geodesic circle $c$ that cuts $A \backslash[p q]$ into more than two pieces. But $c$ cuts A into at most two pieces, thus $[p q]$ cuts one of the two pieces. Therefore $[p q]$ must cut the boundary of A. Hence $[p q]$ is not contained in the interior which is a contradiction.

Using a similar proof, we get the following proposition:

Proposition 18. Let $A$ be a closed subset of $S^{2}$ having TTPP, and $\operatorname{int} A \neq \phi$. Let $p$ and $q$ be any two points in int $A$. If the minimal geodesic segment $[p q] \subset$ int $A$, then $A \backslash[p q]$ has TTPP.

Proposition 19. Let $A$ be a closed subset of $S^{2}$ having TTPP, $B$ be a convex subset of $A$ and both int $A$ and int $B$ are non-empty. If $B \cap \partial A=\phi$, then $A \backslash B$ has STPP.

Proof. Suppose that $A \backslash B$ does not have STPP, then there exists a geodesic circle $c$ that cuts $A \backslash B$ into more than two pieces. But $c$ cuts $A$ into at most two pieces, therefore $B$ intersects one of the two pieces into at least two pieces i.e $B$ must intersect the boundary of $A$ since $B$ is convex. This contradiction completes the proof.

Using a similar proof, we get the following proposition:

Proposition 20. Let $A$ be a closed subset of $S^{2}$ having TTPP, $B$ be a convex subset of $A$ and both int $A$ and int $B$ are non-empty. If $B \cap \partial A=\phi$, then $A \backslash B$ has TTPP.

Remark 21. In the above Proposition if we replace convexity of $B$ by TTPP, then $A \backslash B$ does not neccessarly have TTPP. For example if $A$ is a geodesic ball in $S^{2}$ and $B$ is the area between two concentric geodesic balls in int $A$, then $A \backslash B$ does not have TTPP. 
Theorem 22. Let $A \subset S^{n}$ be a connected open subset contained in an open hemisphere with smooth boundary $\partial A$. If each boundary point $q \in \partial A$ is a local farthest point of $A$ for some point $p$ in $S^{n}$ such that $d(p, q) \prec \pi / 2$. Then A has TPP.

Proof. Let $q \in \partial A$ be an arbitrary point. Then by hypothesis there exists some point $p \in S^{n}$ and a neighborhood $U$ about $q$ such that $d(p, q) \succ d(p, x)$ for all $x \in A \cap U$. Let $B(p, \epsilon), \epsilon=d(p, q)$ be a closed geodesic ball with center $p$ and raduis $\epsilon$. Since $B(p, \epsilon)$ is strictly convex body and so the point $q \in \partial B(p, \epsilon)$ is an exposed point of A with respect to $p$. Therefore there exists a totally geodesic hypersurface $T_{q}$ such that $B(p, \epsilon)$ is contained in one side of $T_{q}$ as in Figure 4. Since $B(p, \epsilon) \cap U \subset B(p, \epsilon), B(p, \epsilon) \cap U$ is also contained in one side of $T_{q}$. Now the geodesic segment $[p q]$ meets $\partial A$ and $\partial B(p, \epsilon)$ orthogonally, which means that A is locally supported at each boundary point. Consequently A is a convex subset of $S^{n}$. Using Lemma 11 we have that A has TTPP.

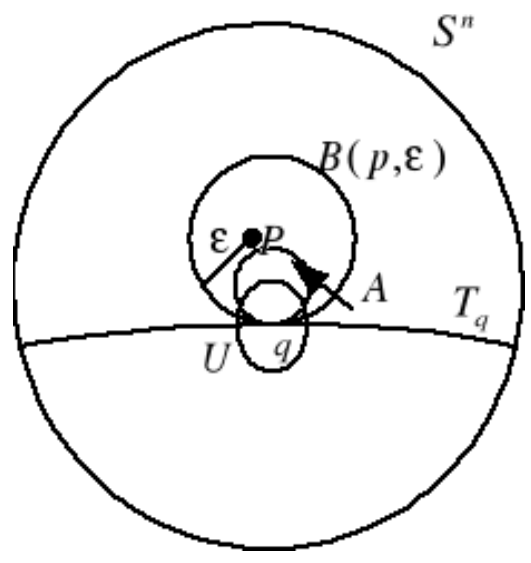

Figure 4

Theorem 23. Suppose that $A$ is a closed subset of $S^{n}$ with smooth boundary and int $A \neq \phi$. If $A$ has STPP, then $A$ is tight.

Proof. First suppose that A is not tight. Then there exists a height function $h_{T}^{p}$ having at least two strict local maximum (say $q$ and $r$ ) (See Figure 5). Either $h_{T}^{p}(q)=h_{T}^{p}(r)$ or $h_{T}^{p}(q) \neq h_{T}^{p}(r)$. For the case $h_{T}^{p}(q)=h_{T}^{p}(r)$ let $S(p, d(p, q))$ be the hypersphere which is tangent to $\mathrm{A}$ at $q$ and $r$ see Figure 5 case 1 . The geodesic sphere $S(p, d(p, q)+\epsilon)$ for sufficiently small $\epsilon \succ 0$ cuts A into more than 
two pieces which is a contradiction. Similar discussion shows that $h_{T}^{p}(q) \neq h_{T}^{p}(r)$ is also impossible see Figure 5 case 2.

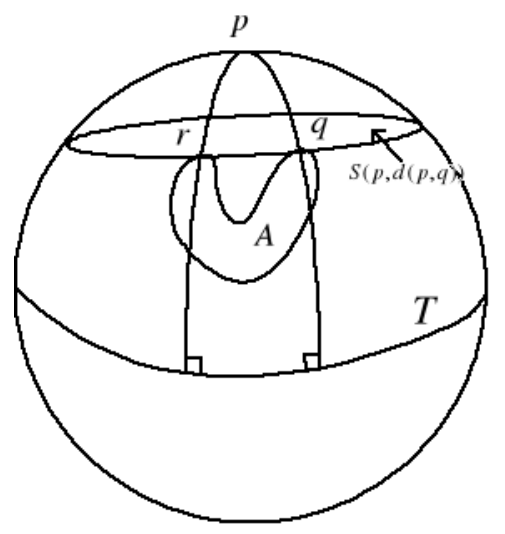

Casel

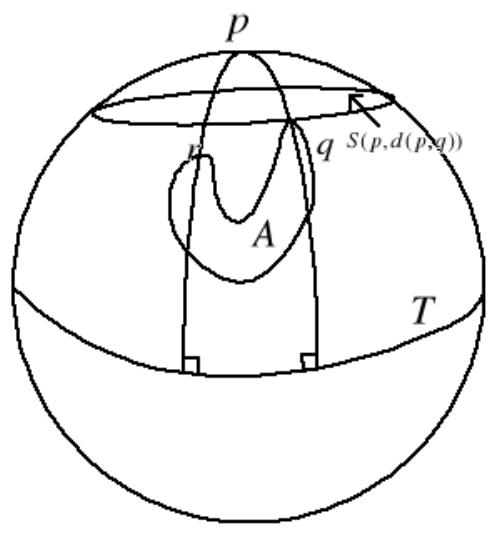

Case 2

Figure 5

Remark 24. 1. The converse of the above theorem is not generally true. For example consider the subset $A \subset S^{2}$ given in Figure 1. A is tight but it does not have STPP.

2. We can find sets which are convex but do not have STPP. For example consider the subset $A \subset S^{2}$ given in Figure 1 with omitted points $p$ and $q$. In this case A is convex, but does not have STPP.

\section{References}

[1] Alexander, S. Local and global convexity in complete Riemannian manifolds, Pacific J. Math., 70(1978), 283-289.

[2] Banchoff. T. F. The spherical two piece property and tight surfaces in spheres J. Diff. Geom. 4 (1970), 193-205

[3] Beltagy, M. Local and global exposed points, Commun. Fac. Sci. Ank. Series $A_{1}, 43(1994), 57-65$.

[4] Beltagy, M. Two piece property in manifolds without focal points, Indian J. pure appi. Math. 17(7):883-889 July, 1986 
[5] Do Carmo, M. P. Riemannian geometry, Birkhauser, Boston, (1992).

[6] S. Carter and A. West, proc. Lond Math. Sco. (3) 25 (1972), 701-20.

[7] T. E. Cecil, and P. J. Ryan, Tight and taut immersions into hyperbolic space J. Lond Math. Sco. (2) 19 (1972), 561-72. 